\title{
CRITERION VALIDITY AND RELIABILITY OF PERCEIVED STRESS SCALE (PSS) IN A STUDENT POPULATION DURING COVID-19 PANDEMIC
}

\author{
Anna Berardi ${ }^{1}$, Ilaria Ruotolo ${ }^{2}$, Giovanni Sellitto ${ }^{2}$, Francescaroberta Panuccio ${ }^{2}$, \\ Antonella Polimeni ${ }^{3}$, Donatella Valente ${ }^{1,4}$, Marco Tofani ${ }^{5}$ and Giovanni Galeoto ${ }^{1,4}$ \\ ${ }^{I}$ Department of Human Neurosciences, Sapienza University of Rome, Italy \\ ${ }^{2}$ Sapienza University of Rome, Italy \\ ${ }^{3}$ Department of Odontostomatological and Maxillo Facial Sciences, Sapienza University, Rome, Italy \\ ${ }^{4}$ IRCSS Neuromed, Via Atinense, 18, 86077 Pozzilli, IS, Italy \\ ${ }^{5}$ Department of Intensive Neurorehabilitation and Robotics, Bambino Gesù Children's Hospital, Rome, Italy
}

\begin{abstract}
BACKGROUND: Because of coronavirus disease 2019 (COVID-19) in Italy, control measures were adopted, such as closure of schools, universities, workplaces, and subsequently lockdown, so everyone's life changed. This is associated with psychological disorders in general public and in particular on students.

OBJECTIVE: To evaluate validity and reliability of the Perceived Stress Scale (PSS) in the assessment of perceived stress of Italian students after the lifestyle changes due to the outbreak of Covid-19.

STUDY DESIGN: Cross-sectional study

METHODS: A mailing list was used for the recruitment of students. Internal consistency was examined through Cronbach's Alpha Coefficient; Criterion validity was evaluated comparing PSS scores to SF-12 values, and Pearson Correlation Coefficient (PCC) was calculated. Correlation analyses were also used to investigate Cross-cultural validity.

RESULTS: The PSS scale was administered to 400 medical and health professionals' students in July 2020. Alpha Coefficient's value was statistically significant, and correlation with the SF-12 health survey was good ( $<<0,01)$. A correlation was found between Perceived Stress and gender, BMI, and also between PSS scores and time spent sitting and exercising under pandemic.

CONCLUSIONS: The Italian version of PSS scale is a valid and reliable instrument to assess Perceived Stress among medical and health professionals' students.
\end{abstract}

\section{KEYWORDS}

Stress, Covid-19, Pandemic, University Students, PSS

\section{INTRODUCTION}

Stress is the nonspecific response of the body to any demand made upon it. (Hans Selye, 1973)

Stress considerably impacts the quality of life and is associated with a range of adverse health outcomes. It can also lead to mental illness, which is a burden for the individual but may also cause serious productivity losses with societal implications. Although a stress condition carries substantial difficulties and limitations, it is merely considered a 'risk factor.' In fact, no diagnosis code for stress exists in the 5th version of the Diagnostic and Statistical Manual of Mental Disorders (DSM V) (M. G. Nielsen et al., 2016; American Psychiatric Association, 2013).

Coronavirus disease 2019 (COVID-19) is a new coronavirus that has never been found in humans before. It spread rapidly worldwide, causing severe acute respiratory syndrome. (Hao Tian et al. 2020) The effective control of SARS-CoV-2/COVID-19 required intensive contact tracing, quarantine of people with suspected infection, and the isolation of infected ones (World Health Organization, 2020). 
The World Health Organization (2020) declared a pandemic state on 11th March 2020 (A. Babore et al., 2020). In Italy, the Civil Protection Department adopted control measures such as information campaigns, closure of schools, universities, and workplaces, and subsequently a lockdown. (F. Gallè et al., 2020) In this critical situation, everyone's life changed due to restrictions to physical movement and social contacts (A. Babore et al., 2020).

According to a recent rapid review on the quarantine effects, society faces several adverse cognitive and emotional problems, such as confusion, poor concentration, irritability, insomnia, distress, frustration, and anger. People are worried about the quarantine duration, insufficient information availability, economic problems, and stigma. These negative effects may affect individuals' bio-psycho-social functioning and lead to depressive or posttraumatic stress symptoms. (S. M. F. Pizzoli et al., 2020) The outbreak of COVID-19 is associated with considerable psychological disorders (PD) in the general public, specific communities, or medical students, especially when the infection rate and deaths are considerable (Hao Tian et al. 2020).

A recent review of the literature of past epidemics and pandemics highlighted that, when comparing the psychological outcomes of quarantined versus non-quarantined people, the first ones show more psychological distress. A study analyzed psychological distress in Italy's general population during the COVID-19 pandemic and showed that the majority of the increase in distress levels noted in this research was related to the course of the pandemic. (C. Mazza et al., 2020) There is evidence of the current pandemic's psychological and mental health effects on students known to be a vulnerable population. (Changwon Son et al., 2020) The mental health of college students is significantly affected when faced with public health emergencies, and they require attention, help, and support from society, families, and colleges (Xiaoyan Liu, Jiaxiu Liu, Xiaoni Zhong, 2020; Hans Selye, 1973).

Several studies conducted on undergraduate students showed that almost half of them decreased their physical activity, and a reduction in physical activity is known to contribute to stress levels (F. Gallè et al., 2020; F. Gallè et al., 2020; Melling Qi et al., 2020).

The Perceived Stress Scale (PSS) is a widely used instrument for measuring stress. The PSS evaluates the degree to which an individual perceives his life as uncontrollable and overloading during the previous month; it also assesses the ability of students to face external demands (M. G. Nielsen et al., 2016). Thus, PSS could be a suitable tool to evaluate Italian students' perceived stress after the lifestyle changes due to Covid-19 pandemic.

In other countries, PSS was already used among students during the lockdown, such as in Turkey, Colombia, Poland, Arabia, Canada (Fuat Torun, Sebahat Dilek Torun, 2020; J. C. Pedrozo-Pupo, M. J. Pedrozo-Cortés, A. Campo-Arias, 2020; Rogowska AM, Kuśnierz C, Bokszczanin A., 2020; Deemah A. AlAteeq, Sumayah Aljhani, Dalal AlEesa, 2020; C. El Morr et al., 2020).

The primary objective of this study is to evaluate the validity and reliability of the PSS in the assessment of perceived stress of Italian students following lifestyle changes due to the outbreak of Covid-19.

\section{METHODS}

A research group of Sapienza University of Rome and Rehabilitation \& Outcome Measures Assessment (ROMA) association conducted a cross-sectional study to evaluate the psychometric properties of PSS (Amedoro A. et al., 2020; Berardi A et al., 2019; Berardi A. et al., 2020; Galeoto G. et al., 2020; Galeoto G. et al., 2019; Ioncoli M et al., 2020; Panuccio F. et al., 2020; Tofani M et al., 2019; Tofani M. et al., 2018).

The institutional review board of Sapienza University of Rome approved the study and guaranteed ethical standards and procedures. Informed consent was asked to all the participants, and they were fully anonymized.

The datasets analyzed during the study are available from the corresponding author.

The inclusion criteria were to be Medical or Health Professionals university students in Italy.

\subsection{Outcome Measures}

The PSS was originally developed as a 14-item scale that assesses the perception of stress by asking the individual to indicate the frequency of feelings and thoughts related to events and situations over the previous month. There are also two short forms, the PSS-4 and PSS-10, with 4 and 10 respectively selected items from the original PSS-14 form. (E. Andreou et al., 2011) PSS-10 scores are obtained by reversing the scores on the 
four positive items 4, 5, 7, and 8 . Total scores range from 0 to 40 , and higher scores indicate greater overall distress. (Müge Çelik Örücü, Ayhan Demir, 2009) The PSS was compared to the SF-12 survey.

The SF-12 is a generic short form health survey developed in the USA from SF-36. It includes two summary measures evaluating physical and mental self-perceived health: Physical Component Summary and Mental Component Summary.

\subsection{Procedures and Data Analysis}

The research group recruited participants according to the inclusion criteria. A mailing list was used to recruit university students attending the faculty of Medicine and Surgery or Health Professions in several Universities in Italy. All participants gave informed consent and then completed the PSS questionnaire.

\subsection{Reliability}

Internal consistency indicates the correlation between different items in the same tool. It was examined through Cronbach's Alpha Coefficient: 0,7 is the significant value recommended to show homogeneity between items.

\subsection{Validity}

Criterion validity was evaluated by comparing PSS scores to SF-12 values. The two assessment tools were administered together, and the Pearson Correlation Coefficient (PCC) was calculated. PCC can be interpreted as follows: 0 indicates no linear relationship; $+1 /-1$ indicates perfect positive/negative linear relationship; between 0 and \pm 0.3 weak relationship; between \pm 0.3 and \pm 0.7 moderate relationship; between \pm 0.7 and \pm 1.0 strong relationship. All statistical analyses were conducted using Statistical Package for Social Sciences (SPSS).

Correlation analysis was used to investigate Cross-cultural validity, in particular between PSS scores and gender of participants, University of origin (North, Central, and South Italy), BMI results, and the total scale scores were also compared to the number of hours spent sitting and exercising during the lockdown.

\section{RESULTS}

The PSS scale was administered to 400 medical and health professionals' students recruited by the Department of Human Neurosciences of Sapienza University of Rome in July 2020, together with the SF-12. The demographic characteristics of the sample are summarized in Table 1.

Table 1. Demographic Characteristics of the sample

\begin{tabular}{|l|l|}
\hline & Mean (SD) \\
\hline Age & $22.9(5.08)$ \\
\hline Body Mass Index & $22.44(4.44)$ \\
\hline $\begin{array}{l}\text { How many hours a day did you spend sitting during } \\
\text { the Lockdown (March 2020-May 2020)? }\end{array}$ & $8.18(3.89)$ \\
\hline $\begin{array}{l}\text { How many hours a day did you exercise during the } \\
\text { Lockdown period (March 2020-May 2020)? }\end{array}$ & $1.65(1.96)$ \\
\hline Gender & Frequency (Percentage) \\
\hline Men & $98(24.5 \%)$ \\
\hline Women & $302(75.5 \%)$ \\
\hline
\end{tabular}

\subsection{Reliability}

Table 2 contains results from an analysis of internal consistency. Alpha Coefficient's value was 0,856 . That is a statistically significant value. 
Table 2. Internal consistency: Cronbach's alpha coefficient

\begin{tabular}{|l|l|l|l|}
\hline & Mean & St. Deviation & Cronbach's Alpha if Item Deleted \\
\hline Item 1 & 1.34 & 1.190 & 0.842 \\
\hline Item 2 & 1.58 & 1.213 & 0.828 \\
\hline Item 3 & 2.53 & 1.090 & 0.835 \\
\hline Item 4 & 1.75 & 1.020 & 0.856 \\
\hline Item 5 & 2.05 & 1.010 & 0.843 \\
\hline Item 6 & 2.22 & 1.155 & 0.840 \\
\hline Item 7 & 2.05 & 0.953 & 0.857 \\
\hline Item 8 & 1.91 & 0.968 & 0.844 \\
\hline Item 9 & 2.05 & 1.081 & 0.843 \\
\hline Item 10 & 1.78 & 1.210 & 0.832 \\
\hline Total Scale & & & 0.856 \\
\hline
\end{tabular}

\subsection{Criterion Validity}

Correlation with the SF-12 health survey was measured using the Pearson Correlation Coefficient analysis, which showed a good linear correlation $(\mathrm{p}<0.01)$ except for the correlation between Stress and Physical Component Summary of SF-12. Pearson values are respectively 0,013 for correlation between PCS12 and PSS, and $-0,390$ for those between MCS12 and PSS, with this one significant at the 0.01 level (2-tailed).

\subsection{Cross Cultural Validity}

A correlation was found between perceived stress and the gender of participants, BMI, and also between PSS scores and time spent sitting and exercising during the pandemic. [Figure 1-4] In particular, time spent sitting is significantly correlated with the BMI of participants and PSS scores, as shown in Table 3.

Table 3. Cross Cultural Validity: Pearson's correlation coefficient

\begin{tabular}{|c|c|c|c|c|c|}
\hline & Gender & Age & $\begin{array}{l}\text { University of } \\
\text { Italy }\end{array}$ & BMI & PSS \\
\hline $\begin{array}{l}\text { How many hours a day did you spend } \\
\text { sitting during the lockdown (March } \\
\text { 2020-May 2020)? }\end{array}$ & $-0.114^{*}$ & $-0.110^{*}$ & -0.003 & $0.129^{* * *}$ & $0.161^{* *}$ \\
\hline $\begin{array}{l}\text { How many hours a day did you } \\
\text { exercise during the lockdown (March } \\
\text { 2020-May 2020)? }\end{array}$ & 0.025 & $-0.104^{*}$ & -0.022 & -0.037 & $-0.112^{*}$ \\
\hline
\end{tabular}




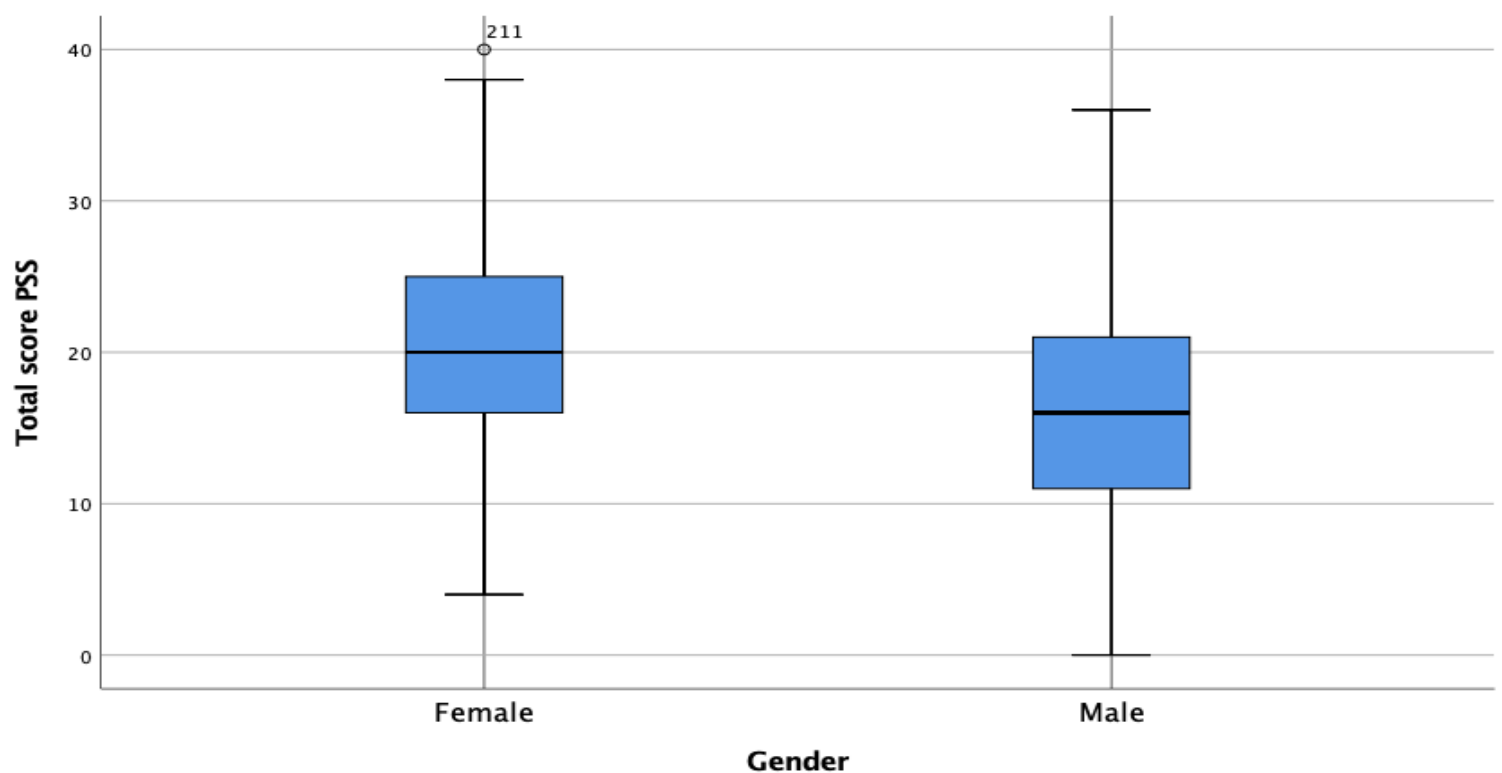

Figure 1. Correlation between gender and PSS total score

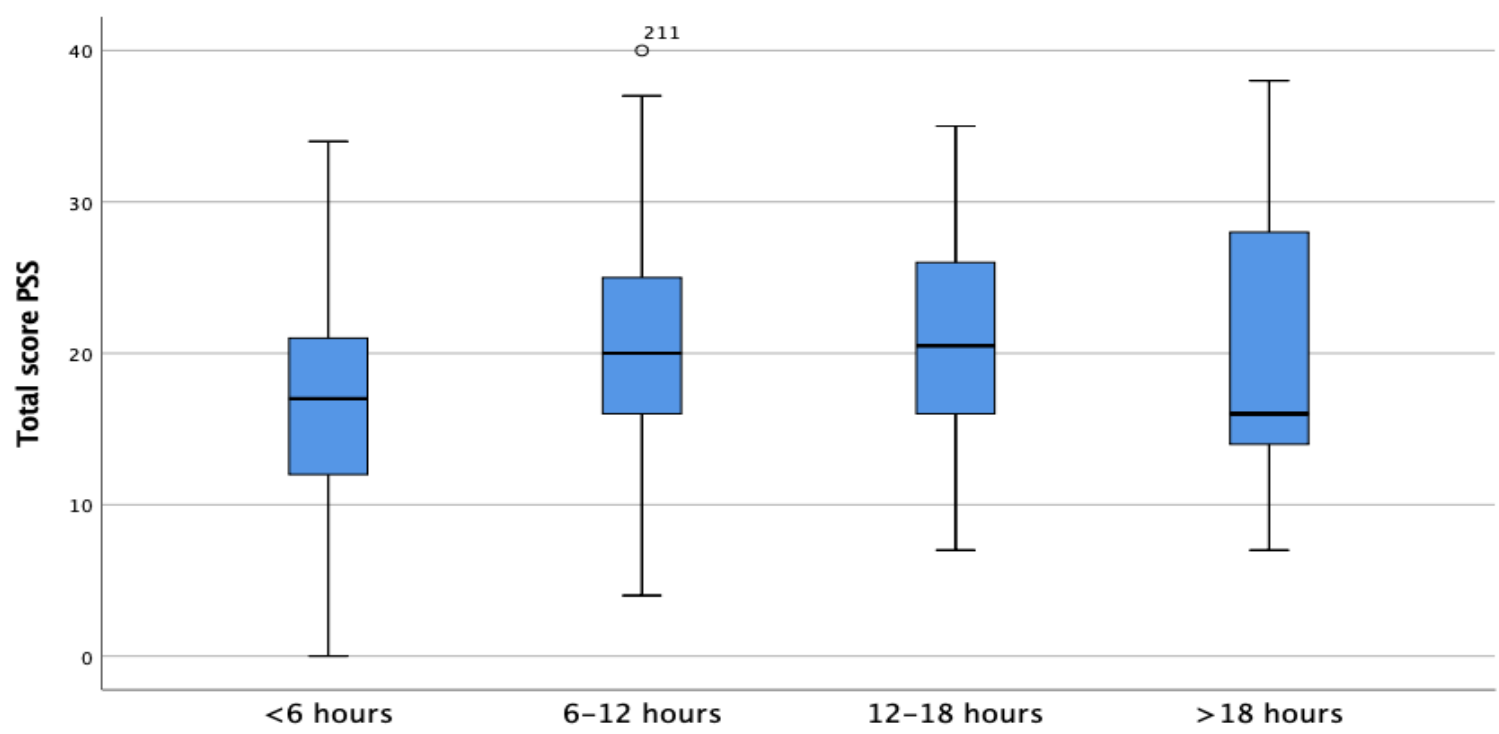

Figure 2. Correlation between time spent sitting and PSS total score 


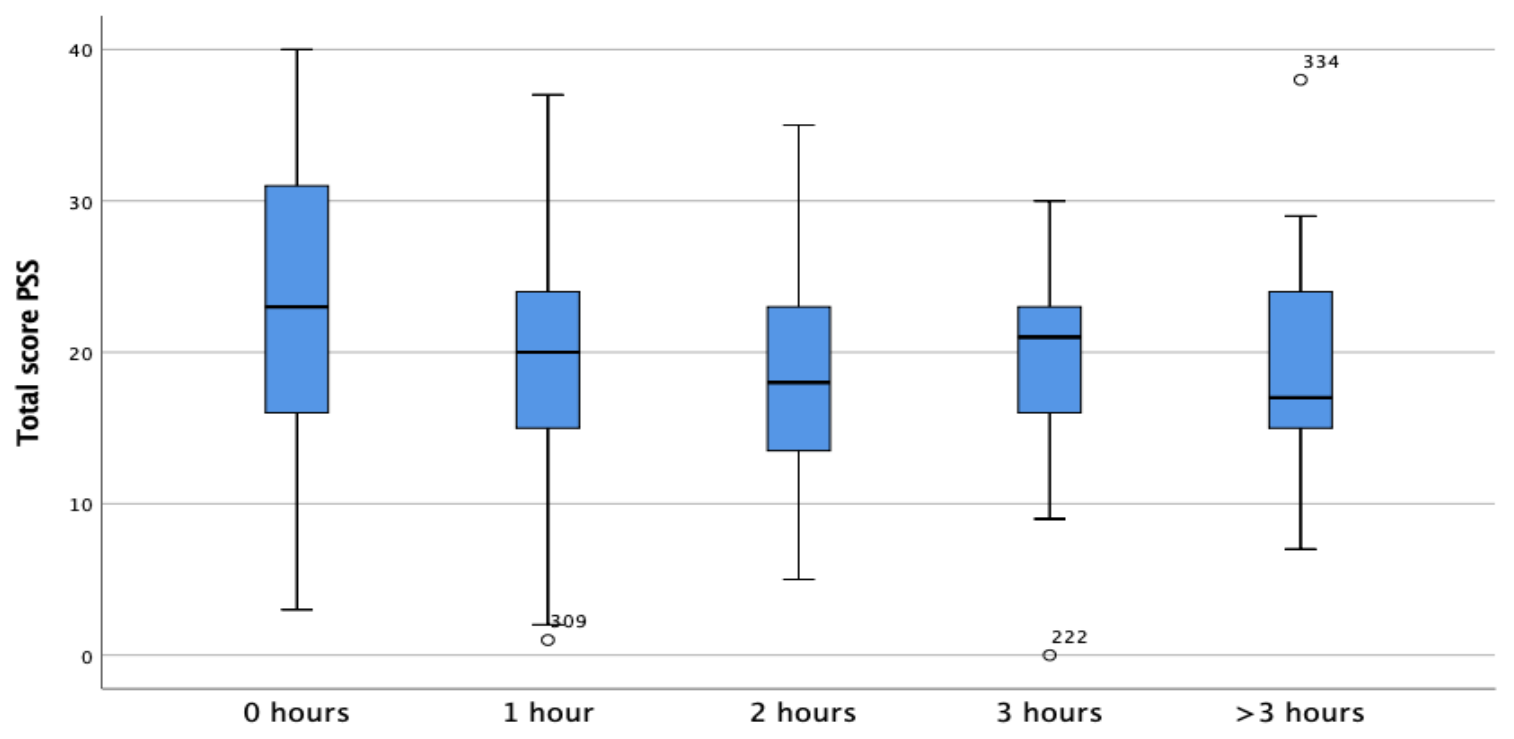

Figure 3. Correlation between time spent exercising and PSS total score

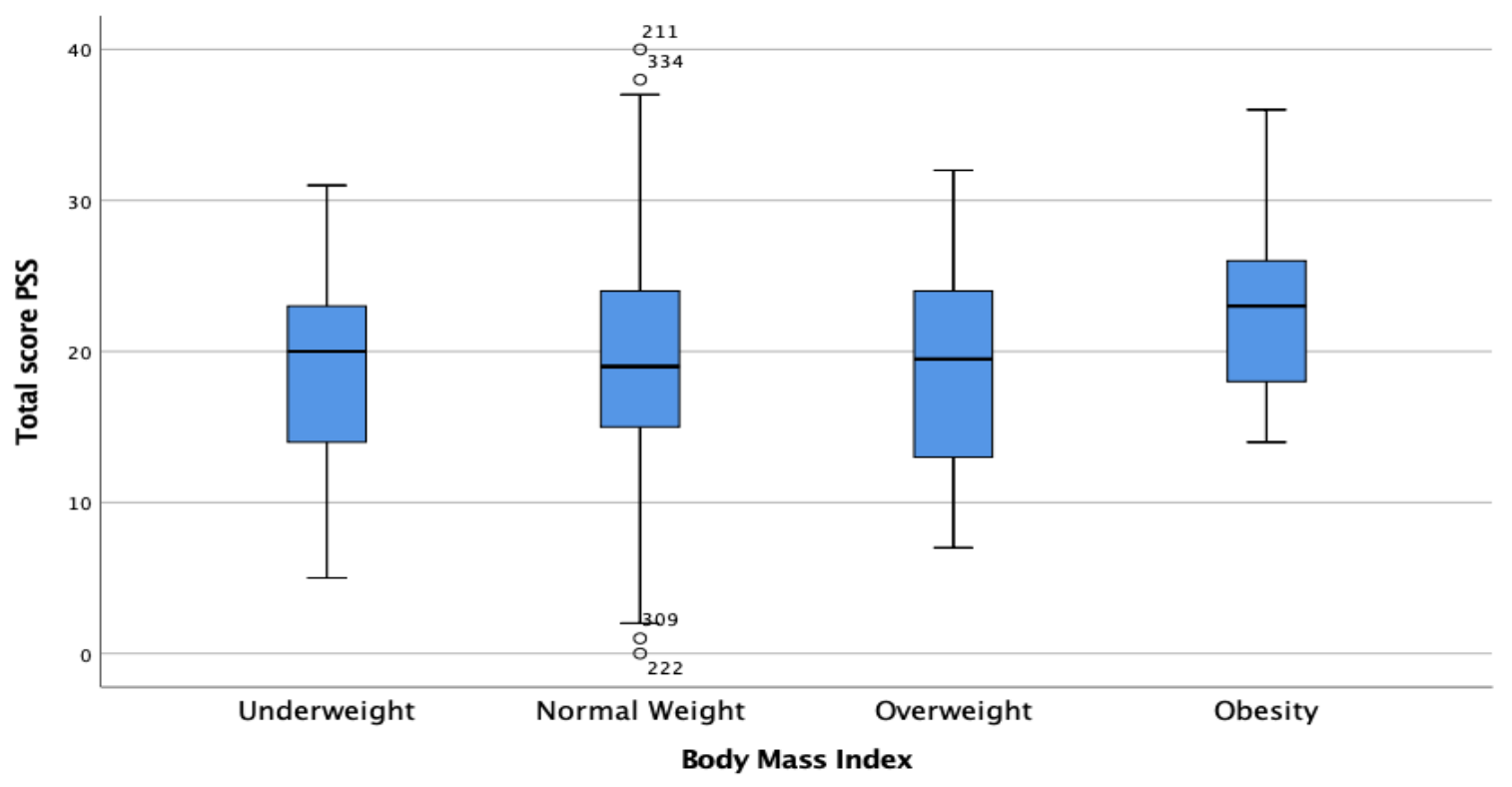

Figure 4. Correlation between BMI and PSS total score

\section{DISCUSSION}

Results show that there is a correlation between PSS scores and the gender of participants. Female students totalized higher scores than males, which means that their distress level was greater than the males. [Figure 1]. Recent studies that investigated the impact of COVID-19 in China have also suggested that gender was a consistent predictor of psychological outcomes. Females were more significantly affected by psychological distress than males who showed moderate anxiety levels (C. Mazza et al., 2020). 


\section{CONCLUSION}

This study showed that the Italian version of the PSS scale is a valid and reliable instrument to assess perceived stress among medical and health professional students.

The authors report no conflict of interest.

\section{REFERENCES}

A. Babore et al., 2020. Psychological effects of the COVID-2019 pandemic: Perceived stress and coping strategies among healthcare professionals. Psychiatry Research, https://doi.org/10.1016/j.psychres.2020.113366

Amedoro A. et al., 2020. The effect of aquatic physical therapy on patients with multiple sclerosis: A systematic review and meta-analysis. Mult Scler Relat Disord.

American Psychiatric Association, 2013. Diagnostic and Statistical Manual of Mental Disorders (DSM-5®), Fifth Edition.

Berardi A et al., 2019. Internal consistency and validity of the Jebsen-Taylor hand function test in an Italian population with hemiparesis. NeuroRehabilitation.

Berardi A. et al., 2020. Tools to assess the quality of life in patients with Parkinson's disease: a systematic review. Expert Review of Pharmacoeconomics and Outcomes Research.

C. El Morr et al., 2020. Effectiveness of an 8-Week Web-Based Mindfulness Virtual Community Intervention for University Students on Symptoms of Stress, Anxiety, and Depression: Randomized Controlled Trial. JMIR Publications.

C. Mazza et al., 2020. A Nationwide Survey of Psychological Distress among Italian People during the COVID-19 Pandemic: Immediate Psychological Responses and Associated Factors. Environmental Research and Public Health, PMID: 32370116 PMCID: PMC7246819 DOI: 10.3390/ijerph17093165

C. Moreno et al. 2020. How mental health care should change as a consequence of the COVID-19 pandemic. The Lancet Psychiatry, DOI:_https://doi.org/10.1016/S2215-0366(20)30307-2

Changwon Son et al., 2020. Effects of COVID-19 on College Students' Mental Health in the United States: Interview Survey Study. JMIR publications, doi: 10.2196/21279 PMCID: PMC7473764 PMID: 32805704

Deemah A. AlAteeq, Sumayah Aljhani, Dalal AlEesa, 2020. Perceived stress among students in virtual classrooms during the COVID-19 outbreak in KSA. Journal of Tibah University Medical Sciences, https://doi.org/10.1016/j.jtumed.2020.07.004

E. Andreou et al., 2011. Perceived Stress Scale: Reliability and Validity Study in Greece. Environmental Research and Public Health, https://doi.org/10.3390/ijerph8083287

F. Gallè et al., 2020. Understanding Knowledge and Behaviors Related to CoViD-19 Epidemic in Italian Undergraduate Students: The EPICO Study. Environmental Research and Public Health, doi: 10.3390/ijerph17103481

F. Gallè et al., 2020. Sedentary Behaviors and Physical Activity of Italian Undergraduate Students during Lockdown at the Time of CoViD-19 Pandemic. Environmental Research and Public Health, https://doi.org/10.3390/ijerph17176171

Fuat Torun, Sebahat Dilek Torun, 2020. The psychological impact of the COVID-19 pandemic on medical students in Turkey. Pakistan Journal of Medical Sciences, PMCID: PMC7501012 PMID: 32968408 doi: 10.12669/pjms.36.6.2985

Galeoto G. et al., 2019. Evaluation of the psychometric properties of the Barthel index in an Italian ischemic stroke population in the acute phase: A cross-sectional study. Funct Neurol.

Galeoto G. et al., 2020 Levels of Cognitive Functioning Assessment Scale: Italian cross-cultural adaptation and validation. Ann di Ig.

Hans Selye, 1973. The Evolution of the Stress Concept: The originator of the concept traces its development from the discovery in 1936 of the alarm reactions to modern therapeutic applications of syntoxic and catatoxic hormones. American Scientist Vol. 61, No. 6

Hao Tian et al. 2020. Humanistic care and psychological counseling on psychological disorders in medical students after COVID-19 outbreak. A protocol of systematic review. Medicine (Baltimore), doi: 10.1097/MD.0000000000021484 PMCID: PMC7437756 PMID: 32871994

Ioncoli $\mathrm{M}$ et al., 2020. Crosscultural Validation of the Community Integration Questionnaire-Revised in an Italian Population. Occup Ther Int.

J. C. Pedrozo-Pupo, M. J. Pedrozo-Cortés, A. Campo-Arias, 2020. Perceived stress associated with COVID-19 epidemic in Colombia: an online survey. Saude Publica, https://doi.org/10.1590/0102-311X00090520

M. G. Nielsen et al., 2016. The construct validity of the Perceived Stress Scale. Journal of Psychosomatic Research, DOI: 10.1016/j.jpsychores.2016.03.009 PMID: 27095155 
Melling Qi et al., (2020). Physical Activity, Health-Related Quality of Life, and Stress among the Chinese Adult Population during the COVID-19 Pandemic. Environmental Research and Public Health, https://doi.org/10.3390/ijerph17186494

Müge Çelik Örücü, Ayhan Demir, 2009. Psychometric evaluation of perceived stress scale for Turkish university students. Stress and Health, https://doi.org/10.1002/smi.1218

Panuccio F. et al., 2020. Development of the Pregnancy and Motherhood Evaluation Questionnaire (PMEQ) for evaluating and measuring the impact of physical disability on pregnancy and the management of motherhood: a pilot study. Disabil Rehabil [Internet]. 4;1-7.

Rogowska AM, Kuśnierz C, Bokszczanin A., 2020. Examining Anxiety, Life Satisfaction, General Health, Stress and Coping Styles During COVID-19 Pandemic in Polish Sample of University Students. Psychology Research and Behavior Management, DOI: https://doi.org/10.2147/PRBM.S266511

S. M. F. Pizzoli et al., 2020. An Internet-Based Intervention to Alleviate Stress During Social Isolation with Guided Relaxation and Meditation: Protocol for a Randomized Controlled Trial. JMIR Research Protocols, DOI: 10.2196/19236 PMCID: PMC7301689 PMID: 32530814

S. M. Skevington, M. Lotfy, K. A. O'Connell, WHOQOL Group, 2004. The World Health Organization's WHOQOL-BREF quality of life assessment: psychometric properties and results of the international field trial. A report from the WHOQOL group. Quality of Life Research, DOI: 10.1023/B:QURE.0000018486.91360.00 PMID: 15085902

Tofani M et al., 2019. Validation of the Pediatric Evaluation of Disability Inventory in an Italian Population with Autism Spectrum Disorder: a Cross-Sectional Study. Clin Ter.

Tofani M. et al., 2018. The psychosocial impact of assistive device scale: Italian validation in a cohort of nonambulant people with neuromotor disorders. Assist Technol.; 25:1-6.

Tofani M. et al., 2019. A study validating the Italian version of the Level of Sitting Scale in children with cerebral palsy. Clin Rehabil.

Tofani M. et al., 2019. The Italian version of rheumatoid arthritis pain scale (IT-RAPS): psychometric properties on community and clinical samples. Reumatismo.

World Health Organization, 2020. Coronavirus disease (COVID-19) pandemic. https://www.who.int/emergencies/diseases/novel-coronavirus-2019

Xiaoyan Liu, Jiaxiu Liu, Xiaoni Zhong, 2020. Psychological State of College Students During COVID-19 Epidemic. The Lancet Global Health.

Yinjian Ge, Shimeng, Xin, Dechun Luan, Zhili Zou, Mengting. Liu, Xue Bai, Qian Gao (2019). Association of physical activity, sedentary time, and sleep duration on the health-related quality of life of college students in Northeast China. Health and Quality of Life Outcomes, DOIhttps://doi.org/10.1186/s12955-019-1194-x 